\title{
Perceived health status in celiac disease
}

\author{
F. Casellas, J. López Vivancos ${ }^{1}$ and J. R. Malagelada \\ Service of Digestive Diseases. Hospital Universitario Vall d'Hebron. ${ }^{1}$ Service of Internal Medicine. Hospital General de \\ Catalunya. Barcelona, Spain
}

\begin{abstract}
Introduction: chronic conditions modify perceived health in affected individuals. For this reason celiac disease, being a chronic condition, may impair health-related quality of life (HRQOL).

Objective: to analyze the impact of celiac disease in affected individuals.

Method: observational, cross-sectional, prospective study in patients with celiac disease by administering two HRQOL questionnaires: EuroQol-5D and GastroIntestinal Quality of Life (GIQLI).

Results: 54 stable patients on a gluten-free diet for a median 60 months, and 9 newly diagnosed individuals still on their usual diet were included. Overall GIQLI score was significantly higher, meaning a better HRQOL, in treated celiac patients versus pretreated celiac patients (3.1 [2.7-3.5] vs. 2.4 [2.1-2.6], $\mathrm{p}<0.01)$. Similarly, EuroQol's health status preference value was also significantly better in treated patients $(0.87[0.8-1.0]$ vs. 0.7 [0.5-0.8], $\mathrm{p}<0.01)$. EuroQol's visual analogic scale had also better scores, representing a better perceived health, among treated patients (80.0 [70.0-90.0] vs. 65.0 [40.0-71.0], p < 0.05). In comparison to EuroQol-5D scores among the healthy Spanish population, values obtained for celiac patients under treatment are similar to those seen in the general population.

Conclusions: celiac disease impairs perceived health in affected individuals, which improves and reaches results similar to those in the general population when on a gluten-free diet.
\end{abstract}

Key words: Celiac disease. Health status. Quality of life. GIQLI. EuroQol-5D.

Casellas F, López Vivancos J, Malagelada JR. Perceived health status in celiac disease. Rev Esp Enferm Dig 2005; 97: 794-804.

Partially funded by a grant C03/C02 from Instituto Carlos III.

Recibido: 21-04-05.

Aceptado: 09-06-05.

Correspondencia: Francesc Casellas. Servicio de Aparato Digestivo. Hospital Universitari Vall d'Hebron Pg. Vall d'Hebron, 119. 08035 Barcelona. Fax. 9348944 56. e-mail: fcasellas@ vhebron.net

\section{INTRODUCTION}

In recent years a significant advance has taken place in the understanding of celiac disease, particularly of its epidemiology, pathophysiology, and diagnosis. However, a less well-developed aspect has been the impact of this disease on health as perceived by affected individuals. Having a chronic disease has multiple impacts, and most relevant among them is the loss or reduction of perceived health, manifested by a limited health-related quality of life (HRQOL). Not surprisingly, chronic gastrointestinal conditions have a negative impact on HRQOL (1).

We lack well-documented data on the HRQOL of celiac patients in our setting. A review of the available literature reveals that, before treatment onset, $66 \%$ of patients report that their perceived quality of life is either moderate or poor (2), which improves following treatment in $77 \%$ of cases (2). In this sense, adherence to a gluten-free diet makes $82 \%$ of patients consider that they reached a good or very good feeling of well-being (3). Since no specific measuring tool is available for celiac disease, most published studies measuring HRQOL in these patients used the generic questionnaire SF-36. With this tool, adherence to a gluten-free diet has been seen to make patients perceive an HRQOL comparable to that of the Canadian, Italian or Irish general population (4-6). However, other studies that also used SF-36 have reported that HRQOL in celiac patients on a gluten-free diet does not reach normality as seen in the Swedish or Italian general population $(7,8)$. HRQOL-determining factors in these patients include female gender (9), compliance with diet (5), and the presence of symptoms or comorbidity $(5,10,11)$.

The need to obtain information on the impact of celiac disease on individuals in our setting, and the reports of inconsistent results on the effect of gluten-free diet on HRQOL have prompted the performance of the current 
study. Its goals include on the one hand the measurement of perceived health in patients at the time of diagnosis, before gluten-free diet onset, and once the disease is under control by means of such diet; on the other hand a collection of data on the impact of disease, and the provision of overall medical care to these patients.

\section{METHOD}

\section{Patients}

Patients diagnosed with celiac disease using current serological and histological criteria (12), and treated as such or as newly diagnosed, were included for 1 year. Patients were stratified into two groups according to their being controlled with a gluten-free diet at the time of inclusion (gluten-free diet group) or their having not yet initiated such diet (pre-treatment group).

\section{Procedure}

All patients included in the study completed a questionnaire on demographic data, current symptoms, medical control, information on the disease, and issues related to the gluten-free diet (information, friendliness, responses to gluten-free food, etc.).

The compliance with the gluten-free diet of patients included in the gluten-free diet group was established using an adaptation of the self-administered questionnaire by Morisky et al. (13). This questionnaire consists of 4 items regarding the extent of treatment compliance, which are completed using a binary scale (yes/no). The first two questions approach unintentional lack of compliance (sometimes I forget my diet/sometimes I do not carefully comply with my diet), and the last two questions deal with intentional lack of compliance (when I feel well I sometimes discontinue my diet/when not feeling well I sometimes discontinue my diet). If either question 3 or 4 is answered in the affirmative, the patient is considered to have voluntarily discontinued his or her diet. If either question 1 or 2 is answered in the affirmative, the patient is considered to have involuntarily neglected or forgotten his or her diet. This questionnaire was originally described for use in the follow-up of medications, and thus has been adapted for application by substituting a gluten-free diet for drugs. In a preliminary study many patients ticked none of the four possible answers, and added in their own "handwriting" that they never forgot about their diet. For this reason, such fifth option has been added to the present protocol, so that patients ticking the option "I never forget about my diet" will be considered good compliers.

\section{Measuring health-related quality of life}

Two generic questionnaires were used for the measurement of HRQOL: the Spanish version of EuroQol$5 \mathrm{D}$, and the Spanish version of Gastrointestinal Quality of Life (GIQLI). Both were selected given the absence of specific questionnaires for celiac disease, and because of their appropriate validation in Spanish.

EuroQol $(14,15)$ has two parts. The first part describes the health status through five dimensions: mobility, personal care, daily-life activities, pain/discomfort, and anxiety/depression. Each of these dimensions has three items in association with three severity levels. Thus, 243 health status descriptions may ensue. In the second part the individual scores his or her health status on a visual analogic scale from 0 (worst health status conceivable) to 100 (best health status conceivable).

GIQLI is a self-administered generic questionnaire to assess HRQOL for gastrointestinal conditions that was originally developed in Germany and then validated into Spanish in 2001 (16). It consists of 36 specific items on gastrointestinal symptoms collected up in 5 dominions: symptoms (19 questions), physical dysfunction (7 questions), emotional dysfunction (5 questions), social dysfunction (4 questions), and treatment effects (1 question), which are to be scored on a scale from 0 to 4 . This questionnaire is assessed according to the average score for the whole tool and each dominion on a scale from 0 (worst HRQOL) to 4 (best HRQOL).

\section{Statistics}

For statistical analysis, patients included in both groups (gluten-free diet and pre-treatment) were independently analyzed. Results are expressed as median and 2575 percentile values. The presence of statistical differences was estimated using Mann-Whitney's univariate analysis for non-parametric variables or Fisher's exact test, when appropriate. For multivariate analyses the Kruskal-Wallis test for range variance was used.

To establish the presence of impaired HRQOL among groups included versus the general population, EuroQol results were compared to the questionnaire's reference standard as obtained from a Spanish general population of 12,245 individuals (17).

\section{RESULTS}

\section{Patients}

Sixty-three patients diagnosed with celiac disease using serological tests and jejunal histology were included; of these, 54 belong to gluten-free diet group and 9 belong to the pre-treatment group. The demographic characteristics of patients at inclusion are listed in table I. No rele- 
Table I. Main characteristics of patients included in the study, as absolute value or median [percentile 25-percentile 75]

\begin{tabular}{lcc}
\hline & Gluten-free diet group & Pre-treatment group \\
\hline $\mathrm{n}$ & 54 & 9 \\
\hline Age & $35[30-54]$ & $41[26-54]$ \\
\hline Female & $40(74 \%)$ & $5(55 \%)$ \\
\hline Non-smoker & $49(91 \%)$ & $9(100 \%)$ \\
\hline Place of residence & & \\
$<100.000$ inhab. & $17(31 \%)$ & $1(11 \%)$ \\
$100.000-500.000$ inhab. & $12(23 \%)$ & 0 \\
$>500.000$ inhab. & $24(46 \%)$ & $8(89 \%)$ \\
\hline Family status & & $5(55 \%)$ \\
Single & $13(24 \%)$ & $4(45 \%)$ \\
Married & $39(72 \%)$ & 0 \\
Widower/widow & $2(4 \%)$ & 0 \\
\hline Education & & $2(22 \%)$ \\
No education & $3(5 \%)$ & $1(11 \%)$ \\
Primary school & $10(18 \%)$ & $6(67)$ \\
Secondary school & $22(42 \%)$ & $7(78 \%)$ \\
College education & $19(35 \%)$ & $2(22 \%)$ \\
\hline Occupational status & & \\
Employee/self-employed & $37(71 \%)$ & \\
Retired/pensionist & $3(5 \%)$ & \\
Housewife & $8(15 \%)$ & \\
Unemployed & $2(4 \%)$ & $3(5 \%$ \\
Others & & \\
\hline
\end{tabular}

vant differences in major demographic characteristics have been seen between both groups of patients. As expected, young adult, non-smoking patients from an urban setting (mostly Barcelona) predominate. Five patients (3 in the diet group, 2 in the pre-treatment group) reported they had first-degree relatives with celiac disease, for a celiac disease rate among family members of $8 \%$.

\section{Celiac disease characteristics}

Table II lists the major characteristics of disease at inclusion. In 31 patients within the diet group (57\%) and in 3 out of 7 patients included in the pre-treatment group the disease had a classic presentation. Median time for symptoms before celiac disease diagnosis was 1 year for both groups.

At inclusion most patients $(81 \%)$ in the gluten-free diet group reported no symptoms, whereas 7 of 9 patients in the pre-treatment group had symptoms, the most common being diarrhea in 6 individuals. Progression time since diagnosis was significantly longer for patients in the diet group than for patients in the pre-treatment group (60 vs. 0 months, $\mathrm{p}<0.001$ ).

The presence of known associated conditions at inclusion was common, and 15 patients in the treatment group
Table II. Main characteristics of celiac disease in patients included in the study. Results are expressed as absolute value or median [percentile 25-percentile 75] ( $\left.{ }^{*}=p<0.001\right)$

\begin{tabular}{lcc}
\hline & Gluten-free diet group & Pre-treatment group \\
\hline $\mathrm{n}$ & 54 & 9 \\
$\begin{array}{l}\text { Presence of symptoms } \\
\text { at inclusion }\end{array}$ & $10(18 \%)$ & $7(78 \%)^{*}$ \\
$\begin{array}{l}\text { Months since diagnosis } \\
\text { Presence of associated } \\
\text { diseases at inclusion }\end{array}$ & $60.0[21.7-135.0]$ & $0 *$ \\
$\begin{array}{l}\text { Months with complaints } \\
\text { before diagnosis }\end{array}$ & $15(28 \%)$ & $3(33 \%)$ \\
\hline
\end{tabular}

(28\%) as well as 3 patients in the pre-treatment group had comorbidities (33\%). Diseases most commonly associated in the treatment group included thyroid conditions in 5 individuals, IgA immune deficiency in 4, and isolated cases of lymphoma, inflammatory myopathy, depression, Crohn's disease, nephrotic syndrome, and scleroderma. Associated conditions in the pre-treatment group included thyroid disease, Sjögren's syndrome, and scleroderma.

\section{Treatment-related aspects}

All patients in the treatment group received a glutenfree diet. Compliance extent, according to criteria by Morisky et al. (13) in 53 patients who correctly responded to this item, was appropriate in 42 patients (79\%). Unintentional lack of compliance was recorded for 9 patients $(17 \%)-3$ out of forgetfulness, 6 from neglect; intentional lack of compliance was seen in $2(4 \%)$ patients who were on an irregular diet because of absent symptoms. Compliance with the diet is consistent with how easy-to-follow patients perceived it was: $13 \%$ considered it was very easy to follow, $79 \%$ thought it was somewhat complicated, and $8 \%$ believed adherence was very difficult. Although suggested in other publications (18), adherence to a gluten-free diet has not been significantly associated with age (34.5 [30.0-47.0] in compliers vs. 40.0 [30.5-55.5] in non-compliers, $\mathrm{p}=$ n.s.) or treatment duration (72.0 [24.0-130.0] months in compliers vs. 48.0 [12.0-144.0] months in non-compliers) or lower education (10 patients among 42 compliers vs. 2 patients among 11 non-compliers had attended at most primary school, p = n.s.).

Interestingly, while $79 \%$ of patients in the treatment group correctly complied with their gluten-free diet, when asked whether they noted any reactions upon the incidental ingestion of gluten-containing food, 29 patients $(57 \%)$ reported some sort of reaction, which suggests potential non-recognized failures in diet adherence. Most common reactions to the ingestion of gluten-containing food included diarrhea $(\mathrm{n}=20)$, abdominal dis- 
tension $(n=4)$, and vomiting $(n=3)$. Only one patient reported the development of allergy in association with the ingestion of gluten-containing food.

Based on patient perceptions, the initiation of a glutenfree diet improved symptoms in all cases except one. Thirty-one patients reported a complete disappearance of symptoms $(57 \%)$, and the rest reported significant $(n=20)$ or moderate $(\mathrm{n}=2)$ improvements.

\section{Health-related quality of life}

Table III shows values obtained for GIQLI's overall scale and 5 individual dimensions. Differences seen between both groups of patients are statistically significant for each dominion, suggesting that perceived HRQOL is better in patients following dietary restrictions versus newly diagnosed individuals in the pre-treatment group, and this applies to both symptom extent and emotional, physical, and social dysfunction.

\section{Table III. Overall and 5-dimensional GIQLI results for the $\mathbf{5 4}$ patients in the treatment group, expressed as median [percentile 25-percentile 75]. For both groups, the better- scored dimension was the one related to treatment, whereas the emotional and physical dimensions obtained the poorest scores}

\begin{tabular}{lcc}
\hline Dimension & Gluten-free diet group & Pre-treatment group \\
\hline Overall & $3.1[2.7-3.5]$ & $2.4[2.1-2.6]$ \\
Symptoms & $3.1[2.8-3.5]$ & $2.5[2.0-2.7]$ \\
Emotional & $2.7[2.2-3.4]^{*}$ & $2.2[1.6-2.6]$ \\
Physical & $3.1[2.3-3.4]$ & $2.1[1.7-2.7]$ \\
Social & $3.5[3.0-4.0]$ & $2.6[2.1-3.1]$ \\
Treatment & $4.0[4.0-4.0]^{*}$ & $3.0[3.0-3.5] \#$ \\
\hline
\end{tabular}

* $=p<0.001$ intragroup, gluten-free diet; $\#=p<0.001$ intragroup, pre-treatment.
In the treated patient group differences in median values for each dimension reached statistical significance $(\mathrm{p}$ $<0.001$ ), with values ranging from 2.7 [2.2-3.4] for emotional involvement to 4 [4.0-4.0] for treatment effects (Table III). In the pre-treatment group GIQLI dimensional values (Table III) ranged from 2.1 [1.7-2.7] for physical dysfunction to 3.0 [3.0-3.5] for treatment effects $(p<$ $0.01)$. Patients in the pre-treatment group had not initiated their gluten-free diet yet, but responded affirmatively the question in the treatment dimension regarding their "feeling let down by treatment effects", as they had already received other drug/dietary treatments. These results suggest that the impact on health at diagnosis is greater for the functioning and emotional dysfunction dominions, whereas impact would focus more exclusively on emotional dysfunction after treatment onset.

The presence of comorbid diseases may be a factor added to celiac disease that would justify a greater involvement of HRQOL. However, GIQLI's overall score did not differ between the 45 patients with comorbid diseases and all 18 individuals with no associated conditions (3.0 [2.7 - 3.4] vs. 3.0 [2.4 - 3.5], respectively, $\mathrm{p}=$ n.s.).

EuroQol-5D results confirm the presence of impaired HRQOL in patients with celiac disease before treatment onset (Fig. 1). Both EuroQol-5D tariff (0.87 [0.8-1.0] vs. 0.7 [0.5-0.8], $\mathrm{p}<0.01)$ and visual analogic scale $(80.0$ [70.0-90.0] vs. 65.0 [40.0-71.0], $\mathrm{p}<0.05)$ received significantly better scores in the diet group versus the pretreatment group, suggesting a better perceived HRQOL in said group.

For reference regarding HRQOL involvement extent in celiac disease EuroQol-5D results as obtained were compared to those reported for a healthy Spanish population sample, where a median tariff of 1.0 and a median visual analogic scale score of 80 were obtained. Even though no statistical analyses may be performed, median

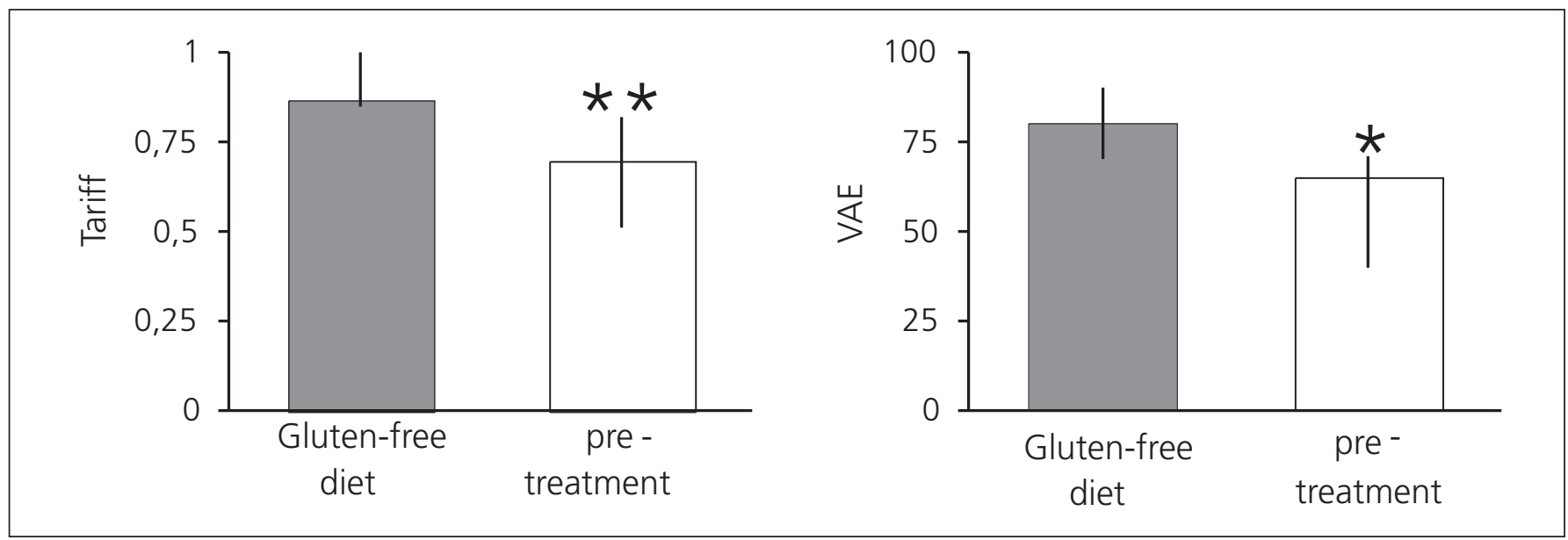

Fig. 1. Median scores for EuroQol-5D tariff (left) and visual analogic scale (right) items for the 54 patients in the treatment group (grey columns) and 9 patients in the pre-treatment group (white columns). Statistical significance levels are below $0.05\left(^{*}\right)$ or $0.01\left(^{* *}\right)$.

Valor de las medianas de las puntuaciones de la tarifa (figura de la izquierda) y de la escala visual analógica (figura de la derecha) del EuroQol-5D para los 54 pacientes del grupo en tratamiento (columnas grises) y los 9 del grupo pretratamiento (columnas blancas). Los niveles de significación estadistica son inferiores a 0,05 (*) o a 0,01 (**). 
tariff and visual analogic scale values for patients under treatment with a gluten-free diet are similar to those of the healthy population, whereas those obtained from patients in the pre-treatment group are lower, which corresponds to a moderate perceived HRQOL.

Despite HRQOL normalization in patients under diet therapy, two subgroups may coexist -one with normalized perceived health and one with persistent involvement. To elucidate this, questionnaire scores were analyzed according to therapy compliance and symptom response. Overall GIQLI scores for all 42 complying patients was no different from those of non-compliers (3.1 [2.7-3.4] vs. 3.0 [2.7-3.5], $\mathrm{p}=$ n.s.), and EuroQol-5D results were also similar between both subgroups (1.0 [0.8$1.0]$ vs. 0.8 [0.8-1.0] y 80.0 [70.0-90.0] vs. 85.0 [72.591.7], respectively). However, overall GIQLI scores were better for all 31 patients considering that symptoms had disappeared with the diet versus all 23 reporting partial improvements only (3.3 [3.0-3.5] vs. 2.8 [2.4-3.1], p < 0.01 , with EuroQol-5D being consistent with such difference (1.0 [0.8-1.0] vs. 0.8 [0.7-1.0], respectively, and 80.0 [76.0-90.0] vs. 70.0 [60.0-85.0], respectively, $\mathrm{p}<$ $0.05)$. The 31 patients who reported that gluten-free food could always be easily found also had better GIQLI scores when compared to all 22 reporting variable difficulty degrees (3.3 [2.9-3.5] vs. 2.8 [2.6-3.3], $\mathrm{p}=0.05)$, but this difference did not reach statistical significance with EuroQol-5D (0.8 [0.7-1.0] vs. 0.9 [0.8-1.0] and 80.0 [70.0-90.0] vs. 80.0 [70.0-86.2], $\mathrm{p}=$ n.s.). HRQOL was not different between the 25 patients who considered themselves well informed regarding appropriate food and the 29 individuals who had concerns or considered themselves insufficiently informed (3.1 [2.6-3.5] vs. 3.2 [2.7$3.4], \mathrm{p}=\mathrm{ns}$ ), with EuroQol-5D also showing no differences between both groups $(0.9$ [0.8-1.0] vs. 0.8 [0.8-1.0] and 80.0 [66.2-88.7] vs. 80.0 [70.0-90.0], respectively, $\mathrm{p}$ $=$ n.s.).

\section{DISCUSSION}

The current study evaluated perceived health in patients with celiac disease using two generic questionnaires to measure HRQOL. Two patient groups were included, one in a baseline condition before treatment onset and one with recognized disease under treatment with a gluten-free diet. Scores for both questionnaires, GIQLI and EuroQol-5D, have been significantly lower in the non-treated group versus the treated group, suggesting a poorer HRQOL in celiac patients at diagnosis. Matching EuroQol-5D scores to those of the Spanish general population reveals that HRQOL status is moderate at the time of diagnosis, but reaches values similar to normal ones in the group already under treatment. The impact of disease on HRQOL affects all dimensions analyzed, particularly the emotional (sadness level, nervousness, fear, etc., regarding disease) and physical (fatigue level, malaise, vi- tality, resistance, etc., regarding disease) dimensions. These results are consistent with the reported experience in other series using also generic tools for HRQOL measurement, and confirm that HRQOL recovers following treatment.

Median scores for GIQLI's treatment dimension reached the scale's maximum in the group of celiac patients under a gluten-free diet. This dimension consists of a single item referring not to treatment efficacy but the fact of feeling more or less upset or let down by their condition's medical treatment. Not surprisingly, scores are pretty high, as diet therapy is well tolerated and has no apparent adverse effects. The treatment dimension received also the best scores in the group of patients who had not initiated their diet yet, which may be accounted for by the fact that many patients have already received symptomatic or replacement therapies with good tolerance. Regarding extent of compliance with the diet, the high percentage of good compliers is noticeable (79\%). This figure is, however, similar to that described in Italian and Swedish studies, which describe appropriate compliance with the gluten-free diet in 73 and $80 \%$ of cases, respectively $(19,20)$.

Given the number of patients included in this study, accurately establishing which factors play a role, and to which extent, in the involvement of HRQOL in celiac disease is a difficult task. The reported experience reveals that the main factors relate to the presence of symptoms or comorbidity $(5,10,11)$, and to diet adherence (5). As is clear from table II, the primary difference between both groups is the presence of symptoms, these being significantly more common in the pre-treatment group. This may lead to consider that higher HRQOL involvement in patients included in the pre-treatment group may be related to presence of symptoms. In support of such association, patients in the treatment group have been seen to have a significantly better HRQOL when compared to those already without symptoms or reporting only a partial improvement of complaints. Nevertheless, symptom improvement is expected to be greater in patients adherent to treatment versus patients who intentionally or unintentionally comply incorrectly with their diet, and so HRQOL is expected to be better in compliers versus noncompliers with the gluten-free diet. However, both GIQLI and EuroQol-5D scores were similar for compliers and non-compliers, suggesting that the crucial factor for HRQOL impairment in celiac patients is the presence of symptoms rather than diet adherence. Further studies are needed with a higher number of patients to fully define these issues.

In the follow-up of celiac disease patients, once a gluten-free diet has been initiated, surveillance to ensure that celiac serology remains negative, and that no clinical symptoms develop is recommended (21). However, as symptoms are heterogeneous in this disease (22), HRQOL measurements may also be included in these patients' follow-up. This would involve patients more ac- 
tively in the control of their disease, which would probably help improve diet-related compliance and adherence.

To conclude, celiac disease significantly impairs HRQOL major dimensions, and health status may be considered moderate. The impact on HRQOL is particularly related to the presence of symptoms. Treatment with a gluten-free diet improves perceived HRQOL to levels matching those of the general population.

\section{REFERENCES}

1. Casellas F, López Vivancos J. Evaluación de la calidad de vida en las enfermedades digestivas. Gastroenterol Hepatol 2004; 27: 58-68.

2. Green PHR, Stavropoulos SN, Panagi SG, Goldstein SL, McMahon DJ, Absan H, et al. Characteristics of adult celiac disease in the USA: results of a national survey. Am J Gastroenterol 2001; 96: 12631.

3. Ciacci C, D'Agate C, De Rosa A, Franzese C, Errichiello S, Gasperi $\mathrm{V}$, et al. Self-related quality of life in celiac disease. Dig Dis Sci 2003; 48: 2216-20.

4. Cranney A, Zarkadas M, Graham ID, Switzer C. The Canadian celiac health survey-the Ottawa chapter pilot. BMC Gastroenterol 2003; 3: 8-14

5. Usai P, Minerba L, Marini B, Cossu R, Spada S, Carpiniello B, et al. Case control study on health-related quality of life in adult coeliac disease. Digest Liver Dis 2002; 34: 547-52.

6. O'Leary C, Wieneke P, Healy M, Cronin C, O'Regan P, Shanahan F. Celiac disease and the transition from childhood to adulthood: a 28year follow-up. Am J Gastroenterol 2004; 99: 2437-41.

7. Hallert C, Grännö C, Grant C, Hultén S, Midhagen G, Ström M, et al. Quality of life of adult coeliac patients treated for 10 years. Scand J Gastroenterol 1998; 33: 933-8.

8. Fera T, Cascio B, Angelini G, Martini S, Sategna C. Affective disorders and quality of life in adult coeliac disease patients on guten-free diet. Eur J Gastroenterol Hepatol 2003; 15: 1287-92.
9. Hallert C, Grännö C, Hultén S, Midhagen G, Ström M, Svensson H, et al. Living with coeliac disease. Controlled study of the burden of illness. Scand J Gastroenterol 2002; 37: 39-42.

10. Mustalahti K, Lohiniemi S, Collin P, Vuolteenaho N, Mäki M. Gluten-free diet and quality of life in patients with screen-detected celiac disease. Eff Clin Pract 2002; 5: 105-13.

11. Johnston SD, Rodgers C, Watson RGP. Quality of life in screen-detected and typical coeliac disease and the effect of excluding dietary gluten. Eur J Gastroenterol Hepatol 2004; 16: 1281-6.

12. NIH Consensus Development Conference Statement: Celiac Disease. Available at: http://consensus.nih.gov/cons/118/118celiacPDF.pdf. Del 30 de septiembre de 2004.

13. Morisky DE, Green LW, Levine DM. Concurrent and predictive validity of a self-reported measure of medication adherence. Med Care 1986; 24: 67-74.

14. EuroQol Group. EuroQol-A new facility for the measurement of health related quality of life. Health Policy 1990; 16: 199-208.

15. Badia X, Fernández E, Segura A. Influence of socio-demographic and health status variables on evaluation of health states in a Spanish population. Eur J Public Health 1995; 5: 87-93

16. Quintana JM, Cabriada J, López I, Varona M, Oribe V, Barrios B, et al. Traducción y validación del Indice de Calidad de Vida Gastrointestinal (GIQLI). Rev Esp Enferm Dig 2001; 93: 693-9.

17. Badia X, Roset M, Montserrat S, Herdman M, Segura A. La versión española del EuroQol: descripción y aplicaciones. Med Clin 1999; 112 (Supl. 1): 79-86.

18. Ciacci C, Cirillo M, Cavallaro R, Mazzacca G. Long-term follow-up of celiac adults on gluten-free diet: Prevalence and correlates of intestinal damage. Digestion 2002; 66: 178-85.

19. Greco L, Mayer M, Cicarelli G, Troncone R, Auricchio S. Compliance to a gluten-free diet in adolescents, or "what do 300 coeliac adolescents eat every day?". Ital J Gastroenterol Hepatol 1997; 29: $305-10$.

20. Hogberg L, Grodzinsky E, Stenhammar L. Better dietary compliance in patients with celiac disease diagnosed in early childhood. Scand J Gastroenterol 2003; 38: 751-4.

21. Pietzak MM. Follow-up of patients with celiac disease: achieving compliance with treatment. Gastroenterology 2005; 128: S135-S141.

22. Green PHR. The many faces of celiac disease: clinical presentation of celiac disease in the adult population. Gastroenterology 2005; 128 : S74-S78 